\title{
Same-day discharge vs. observation after laparoscopic pediatric appendectomy: a prospective cohort study
}

\author{
Kristin M. Gee ${ }^{1}$, Sandra Ngo ${ }^{1}$, Lorrie Burkhalter ${ }^{2}$, Alana L. Beres ${ }^{3}$ \\ ${ }^{1}$ Division of Pediatric Surgery, Department of Surgery, University of Texas Southwestern Medical Center, Dallas, TX, USA; ${ }^{2}$ Children's Health, \\ Dallas, TX, USA; ${ }^{3}$ Division of Pediatric General, Thoracic and Fetal Surgery, University of California Davis, Sacramento, CA, USA \\ Contributions: (I) Conception and design: AL Beres; (II) Administrative support: L Burkhalter; (III) Provision of study materials or patients: L \\ Burkhalter; (IV) Collection and assembly of data: KM Gee, S Ngo; (V) Data analysis and interpretation: S Ngo, KM Gee; (VI) Manuscript writing: \\ All authors; (VII) Final approval of manuscript: All authors. \\ Correspondence to: Kristin M. Gee, MD. Division of Pediatric Surgery, Department of Surgery, University of Texas Southwestern Medical Center, \\ 5323 Harry Hines Blvd., Dallas, TX 75390, USA. Email: Kristin.m.gee@gmail.com.
}

Background: Through 2015, the practice at our university based free-standing children's hospital was to admit uncomplicated appendicitis patients for overnight observation post-operatively. Given the increasing body of evidence suggesting the safety and feasibility of same-day discharge after appendectomy for uncomplicated appendicitis, we elected to perform a prospective study evaluating the complication rates of same-day discharge compared to overnight observation at our institution, given our large volume of appendicitis. Methods: Pediatric patients who underwent laparoscopic appendectomies for uncomplicated appendicitis in 2016 were analyzed. Data regarding demographics, admission, and discharge times and outcomes of complications, as well as readmissions, return to the emergency department, and nonscheduled clinic visits were collected and analyzing using chi-square and multivariate regression. Cost of stay data was obtained and analyzed using Mann-Whitney U test to compare non-parametric variables.

Results: Eight hundred and forty-nine laparoscopic appendectomies were performed for uncomplicated appendicitis during the study period, of which 382 resulted in same-day discharge and 467 in an admission for observation. Univariate analysis revealed no statistical difference between readmission rates for same day vs. observation ( 2 vs. 6 patients; $\mathrm{P}=0.21$ ) or in emergency department visits within 30 days (22 vs. 27 patients; $\mathrm{P}=0.98)$. There was no difference in the number of surgical site infections or extra clinic visits. There was a significantly lower median cost of stay for patients discharged home the same day at 29,150 dollars $(25,644$, $32,276, \mathrm{IQR})$ compared to a median of 34,827 dollars $(31,154,39,457$, IQR $(\mathrm{P}<0.0001)$.

Conclusions: Same-day discharge for laparoscopic uncomplicated appendectomy should be the new standard of care. This study found no differences in outcomes between the timing of discharge, with a significantly lower cost of stay for patients discharged home the same day.

Keywords: Same-day discharge; appendectomy; pediatric; ambulatory surgical procedure; appendicitis

Received: 13 January 2020; Accepted: 21 May 2020; Published: 25 July 2021.

doi: $10.21037 /$ tgh-20-39

View this article at: http://dx.doi.org/10.21037/tgh-20-39

\section{Introduction}

Appendicitis remains the most frequent pediatric emergency requiring surgery $(1,2)$. In a review of 1.9 million discharges of pediatric patients in 1997, appendicitis was the leading diagnosis in terms of total number of hospital days for a gastrointestinal disorder and amassed $\$ 680.4$ million in charges that year (1). However, since the wide adoption of laparoscopic techniques, there has been a decreased incidence of infection, postoperative pain, and length of hospital stay $(3,4)$. Additionally, recent studies have supported the possibility of same-day discharge for both 
adult and pediatric patients with uncomplicated appendicitis who undergo a laparoscopic appendectomy $(5,6)$. A 2012 prospective study of 207 children who were discharged home the same day after uncomplicated or interval appendectomy found a similar complication rate when compared to 45 children who were observed overnight (7). This further supports other retrospective studies with smaller cohorts of adult patients who had similar rates of complications after uncomplicated laparoscopic appendectomy followed by same-day discharge or observation (8). Our institution recently examined the safety and feasibility of same-day discharge after laparoscopic appendectomy at our institution, and found low rates of complications and emergency department visits (9).

Through 2015, Children's Health routinely admitted patients for overnight observation after laparoscopic appendectomy for uncomplicated appendicitis. These patients' length of admission ranged from 8 to over 24 hours for the same procedure. After initial study results demonstrated the safety and feasibility of a sameday discharge plan, we sought to prospectively compare post-operative outcomes and costs of pediatric patients with uncomplicated acute appendicitis based on same-day discharge $v s$. overnight observation.

Our hypothesis was that there would be no significant difference in complication rates between patients discharged on the same day as their surgery compared to patients observed overnight, with potential for significant cost reduction. We present the following article in accordance with the STROBE reporting checklist (available at http:// dx.doi.org/10.21037/tgh-20-39).

\section{Methods}

After institutional review board approval (STU 082015081), all pediatric patients who underwent a laparoscopic appendectomy for uncomplicated appendicitis in 2016 were analyzed. Patients who had interval appendectomies, or surgery for perforated or gangrenous appendicitis were excluded from the study. Patients who were found to have additional intra-abdominal pathology (i.e., Meckel's diverticulum) at the time of surgery were also excluded. The entire surgical division, including the 10 faculty surgeons and 2 fellows, agreed to participate in the study. Decision for same-day discharge was based on surgeon preference and parental agreement. All patients with a diagnosis of uncomplicated appendicitis follow a pre-operative pathway with standard antibiotics (ceftriaxone and metronidazole with 24-hour dosing, or clindamycin and gentamicin if a penicillin allergy). Post-operatively no antibiotics are administered, and there is a protocol pathway with an order set in our electronic medical record. Pain, diet and fluid regimens are standardized. All patients are discharged home with only acetaminophen and ibuprofen and without any opiates. Patients were considered to be same-day discharge if they were sent home prior to 11:59 p.m. on the day of surgery. Patients were either discharged from an inpatient unit if they had been admitted the previous night or from the post-anesthesia care unit (PACU). For the purpose of this study, all patients discharged from the PACU after 11:59 p.m. were not included as a same-day discharge. All data regarding demographics, including gender, age, race and discharge location were recorded. Data regarding admission and discharge times and locations, as well as outcomes of complications, readmissions, return to the emergency department, and nonscheduled clinic visits were collected and analyzed using chi-square and multivariate regression. Both cohorts of patients (same day and observation) received the same set of discharge instructions. Follow-up consisted of our standard phone call at 2 weeks postoperatively. This phone call was used to assess patient's pain, healing, appetite, and return to normal activities. All patients were offered the option of a clinic visit instead of a phone call follow-up. Patient electronic medical records were assessed through the end of January 2017 to evaluate for parent phone calls, readmission to hospital, or emergency department visits.

Univariate analysis (chi-square or Fisher's exact) was used to compare outcomes for same day vs. non-same day. Cost of stay data was obtained for both cohorts and analyzed using Mann Whitney U. Multivariate logistic regression was then used to control for age, gender and discharge from $\mathrm{PACU} v$ s. inpatient floor; with a $\mathrm{P}$ value of $<0.05$ considered significant. Data analysis was performed using Stata 14 (StataCorp LLC, https://www.stata.com/).

\section{Results}

All patients were reviewed for outcomes between January and December of 2016. A total of 1,321 appendectomies were performed during the study period; 849 were uncomplicated, of which $382(40 \%)$ resulted in same-day discharge and $467(60 \%)$ in overnight observation. The same-day discharge patients were either sent home from the PACU (36\%) or from an inpatient unit (64\%). Discharge from the PACU occurred at a median time of 2.4 hours 
Table 1 Demographics for pediatric patients who had either same-day discharge or overnight observation after laparoscopic appendectomy

\begin{tabular}{lcc}
\hline Demographics & Same day, $\mathrm{n}[\%]$ & Observation, $\mathrm{n}[\%]$ \\
\hline Sex & $147[38]$ & $187[40]$ \\
Female & & \\
Age (years) & $41[11]$ & $69[15]$ \\
$0-6$ & $216[57]$ & $264[57]$ \\
$7-12$ & $125[32]$ & $134[28]$ \\
$13-18$ & & \\
Race & $256[68]$ & $312[67]$ \\
Caucasian/white & $21[5]$ & $35[8]$ \\
African American/black & $9[3]$ & $8[2]$ \\
Asian & $37[9]$ & $35[8]$ \\
Hispanic & $38[10]$ & $57[12]$ \\
Other & $21[5]$ & $20[4]$ \\
Unknown & & 0 \\
Discharge location & $139[36]$ & $467[100]$ \\
PACU & $243[64]$ & \\
Floor & & \\
\hline
\end{tabular}

PACU, post-anesthesia care unit.

Table 2 Outcomes for pediatric patients who had either same-day discharge or overnight observation after laparoscopic appendectomy

\begin{tabular}{lccc}
\hline Outcomes & Same day & Observation & P value \\
\hline Abscess & 1 & 2 & 0.575 \\
Nausea/vomiting & 10 & 9 & 0.499 \\
Pain control & 31 & 26 & 0.140 \\
Surgical site & 4 & 9 & 0.226 \\
infection & 2 & & \\
Readmission & 22 & 6 & 0.219 \\
ED visit & 21 & 27 & 0.983 \\
Extra clinic visit & & 15 & 0.139 \\
\hline
\end{tabular}

$(2.18,2.85)$ following surgery and discharge from the floor occurred at a median of 8.1 hours $(6.35,9.88)$ for sameday discharge patients. Observed patients were discharged 21.75 hours $(17.65,26.15)$ after surgery. Follow up (via phone call or clinic visit) was achieved with 257 (67\%)

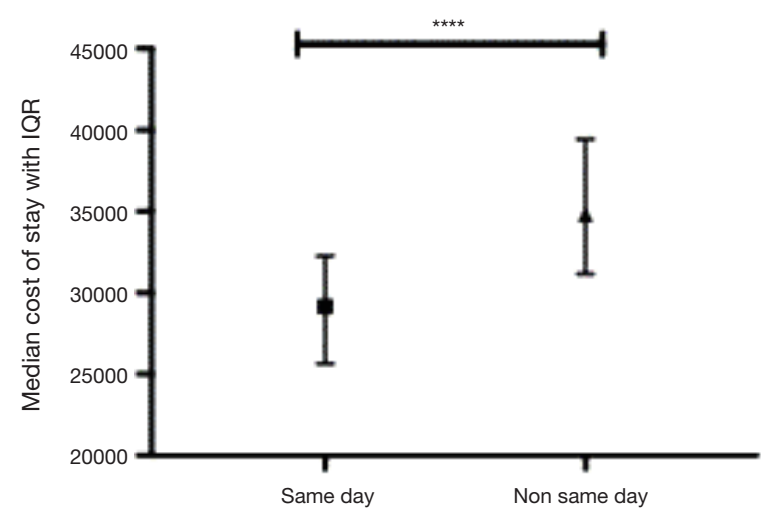

Figure 1 Median cost of stay for same-day discharge $v s$. overnight observation. ${ }^{* * *}, \mathrm{P}<0.0001$.

patients discharged same day and $283(61 \%)$ patients in the observation group, with median follow up of 2 weeks [2,3] for both cohorts.

There was no significant difference in gender, age, or race between the cohorts (Table 1). Univariate analysis revealed no difference between 30 -day readmission rates for same day vs. observation ( 2 vs. 6 patients; $\mathrm{P}=0.21$ ) or in emergency department visits (22 vs. 27 patients; $\mathrm{P}=0.98$ ). There was no difference between the number of surgical site infections ( 4 vs. 9 patients; $\mathrm{P}=0.22$ ) or the number of patients who requested an extra clinic visit (21 vs. 15 patients; $\mathrm{P}=0.13)$, nor a difference in the number of intraabdominal abscesses ( 1 vs. 2 patients; $\mathrm{P}=0.58$ ) (Table 2).

There was a significantly lower median cost of stay for patients discharged home the same day at 29,150 dollars $[25,644,32,276]$ compared to 34,827 dollars $[31,154$, $39,457](\mathrm{P}<0.0001)$ (Figure 1).

On multivariate logistic regression, controlling for age, gender, and discharge from PACU vs. floor, there was a significant difference only for calls related to pain, with the parents of patients who went home the same day slightly more likely to call for pain related issues $(\mathrm{OR}=0.88$; $\mathrm{P}=0.008$ ).

\section{Discussion}

There has been a trend towards same-day discharge after routine laparoscopic procedures for the last decade (10). A 2011 retrospective review of more than 40,000 adult patients comparing laparoscopic to open appendectomy showed lower mortality and morbidity and shorter length of hospital stay for laparoscopic appendectomy patients (11). This 
was further characterized in previous studies that showed the laparoscopic approach is safe for both uncomplicated and complicated appendicitis (12). Our institution as well as many others have previously demonstrated that it is safe to send pediatric patients home the same day after uncomplicated laparoscopic appendectomy with low incidence of negative outcomes such as 30-day readmissions $(9,13,14)$. A 2009 analysis reviewing a protocol for sameday discharge after laparoscopic appendectomy with 72 patients found an average savings of $\$ 323$ per patient (15). In addition to the significant measurable cost savings in our same-day discharge cohort, there are also nonmeasurable cost savings including a reduced parental need to find childcare for other children and to request time off from work. However, given the large cohort of observation patients, it is important to note that both sameday discharge and observation patients have comparably favorable outcomes.

In this prospective comparison study of 849 pediatric patients, we had low readmission rates in both the sameday and observation groups $(0.5 \%$ vs. $1 \%)$. We also had a similar number of post-operative emergency department visits in each group, 22 vs. 27 patients. When examining the 22 emergency department visits in the same-day cohort and combining them with the cohort's additional clinic visits, we found 4 patients with a surgical site infection, 10 with nausea or vomiting, and 33 with pain control issues. We compared that to the 27 emergency visits in the observation group and the group's 15 additional clinic visits and found 9 patients with surgical site infections, 9 with nausea or vomiting, and 26 patients with pain control issues. On multivariate logistic regression, there was no difference between these groups for any of the above complications. In fact, the only significant difference was for calls to the nurse practitioner telephone line related to pain, favoring those who went home the same day $(\mathrm{OR}=0.88 ; \mathrm{P}=0.008)$. This study clearly demonstrates a low and comparable incidence of complications in both groups in contrast to a 2014 study where same-day discharge was associated with higher readmission rates (16). This study's results are also on par with a similar 2014 study showing a readmission rate of $0.7 \%$ when looking at 128 pediatric patients after laparoscopic appendectomy. Additionally, a more recent 2017 study found no statistical difference between sameday and observation groups in regards to ED visits, readmissions, and reoperations $(17,18)$. As the study period progressed, larger numbers of patients were discharged same-day as more physicians in the practice embraced the protocol.

Through the end of 2015 our institution continued to admit all of the routine laparoscopic appendectomies for overnight observation. Additionally, many of the patients were continuing to follow up with an in-office clinic visit at 2 weeks postoperatively instead of a follow-up phone call. Given the large volume of appendectomies performed and the diverse patient population with different levels of access to care, standardizing a protocol for same-day discharges has the potential to greatly decrease cost, improve efficiency, and potentially improve access for follow-up assessment, which has been documented in similar studies (18). Although patient charts were reviewed through January 2017, this study was limited to patients that were reached for follow-up by phone call or seen in clinic, the ED, or during readmission at Children's Health, and it is possible patients presenting to outside hospitals could not be accounted for. Additionally, we acknowledge the inherent bias present in allowing each attending to decide if a patient was suitable for same-day discharge.

\section{Conclusions}

This study shows comparable outcomes for same-day discharge and overnight observation in pediatric patients undergoing routine laparoscopic appendectomy for uncomplicated appendicitis with a significantly reduced cost of stay for the same day admission cohort. Several smaller studies have shown similar results, and so this study with the largest prospective patient cohort contributes to the growing evidence regarding the safety, feasibility, and costeffectiveness of same-day discharge.

\section{Acknowledgments}

The authors would like to thank Dave Primm for his help in editing this manuscript.

Funding: None.

\section{Footnote}

Provenance and Peer Review: This article was commissioned by the Guest Editors (Eduardo Perez, Samir Pandya, and Matthew S. Clifton) for the series "Current Topics in Pediatric General Surgery" published in Translational Gastroenterology and Hepatology. The article has undergone 
external peer review.

Reporting Checklist: The authors have completed the STROBE reporting checklist. Available at http://dx.doi. org/10.21037/tgh-20-39

Data Sharing Statement: Available at http://dx.doi. org/10.21037/tgh-20-39

Conflicts of Interest: All authors have completed the ICMJE uniform disclosure form (available at http://dx.doi. org/10.21037/tgh-20-39). The series "Current Topics in Pediatric General Surgery" was commissioned by the editorial office without any funding or sponsorship. The authors have no other conflicts of interest to declare.

Ethical Statement: The authors are accountable for all aspects of the work in ensuring that questions related to the accuracy or integrity of any part of the work are appropriately investigated and resolved. The study was conducted in accordance with the Declaration of Helsinki (as revised in 2013). This research was approved by our IRB (IRB number: STU 082015-081). The informed consent was waived due to the retrospective nature of the study.

Open Access Statement: This is an Open Access article distributed in accordance with the Creative Commons Attribution-NonCommercial-NoDerivs 4.0 International License (CC BY-NC-ND 4.0), which permits the noncommercial replication and distribution of the article with the strict proviso that no changes or edits are made and the original work is properly cited (including links to both the formal publication through the relevant DOI and the license). See: https://creativecommons.org/licenses/by-nc-nd/4.0/.

\section{References}

1. Guthery SL, Hutchings C, Dean JM, et al. National estimates of hospital utilization by children with gastrointestinal disorders: analysis of the 1997 kids' inpatient database. J Pediatr 2004;144:589-94.

2. McCollough M, Sharieff GQ. Abdominal surgical emergencies in infants and young children. Emerg Med Clin North Am 2003;21:909-35.

3. Sauerland S, Jaschinski T, Neugebauer EA. Laparoscopic versus open surgery for suspected appendicitis. Cochrane Database Syst Rev 2010;(10):CD001546.
4. Aziz O, Athanasiou T, Tekkis PP, et al. Laparoscopic versus open appendectomy in children: a meta-analysis. Ann Surg 2006;243:17-27.

5. Scott A, Shekherdimian S, Rouch JD, et al. Sameday discharge in laparoscopic acute non-perforated appendectomy. J Am Coll Surg 2017;224:43-8.

6. Litz CN, Stone L, Alessi R, et al. Impact of outpatient management following appendectomy for acute appendicitis: An ACS NSQIP-P analysis. J Pediatr Surg 2018;53:625-8.

7. Alkhoury F, Malvezzi L, Knight CG, et al. Routine sameday discharge after acute or interval appendectomy in children: a prospective study. Arch Surg 2012;147:443-6.

8. Cash CL, Frazee RC, Smith RW, et al. Outpatient laparoscopic appendectomy for acute appendicitis. Am Surg 2012;78:213-5.

9. Gee K, Ngo S, Burkhalter L, et al. Safety and feasibility of same-day discharge for uncomplicated appendicitis: a prospective cohort study. J Pediatr Surg 2018;53:988-90.

10. Gould JL, Poola AS, St Peter SD, et al. Same day discharge protocol implementation trends in laparoscopic cholecystectomy in pediatric patients. J Pediatr Surg 2016;51:1936-8.

11. Tiwari MM, Reynoso JF, Tsang AW, et al. Comparison of outcomes of laparoscopic and open appendectomy in management of uncomplicated and complicated appendicitis. Ann Surg 2011;254:927-32.

12. Bensard DD, Hendrickson RJ, Fyffe CJ, et al. Early discharge following laparoscopic appendectomy in children utilizing an evidence-based clinical pathway. J Laparoendosc Adv Surg Tech A 2009;19 Suppl 1:S81-6.

13. Grewal H, Sweat J, Vazquez WD. Laparoscopic appendectomy in children can be done as a fast-track or same-day surgery. JSLS 2004;8:151-4.

14. Halter JM, Mallory B, Neilson IR, et al. Same-day discharge following laparoscopic appendectomy for uncomplicated acute appendicitis as a measure of quality in the pediatric population. J Laparoendosc Adv Surg Tech A 2016;26:309-13.

15. Dubois L, Vogt KN, Davies W, et al. Impact of an outpatient appendectomy protocol on clinical outcomes and cost: a case-control study. J Am Coll Surg 2010;211:731-7.

16. Putnam LR, Levy SM, Johnson E, et al. Impact of a 24-hour discharge pathway on outcomes of pediatric appendectomy. Surgery 2014;156:455-61.

17. Aguayo P, Alemayehu H, Desai AA, et al. Initial experience 
with same day discharge after laparoscopic appendectomy for nonperforated appendicitis. J Surg Res 2014;190:93-7.

18. Yu YR, Smith CM, Ceyanes KK, et al. A prospective same

doi: $10.21037 /$ tgh-20-39

Cite this article as: Gee KM, Ngo S, Burkhalter L, Beres AL. Same-day discharge vs. observation after laparoscopic pediatric appendectomy: a prospective cohort study. Transl Gastroenterol Hepatol 2021;6:45. day discharge protocol for pediatric appendicitis: adding value to a common surgical condition. J Pediatr Surg 2017. doi: 10.1016/j.jpedsurg.2017.10.011. 